\title{
Laptops Educacionais em Escolas Públicas: Primeiros Resultados de uma Abordagem Socio-Técnica
}

Title: Educational Laptops in Public Schools: First Results of an socio-technical approach

Flávia Linhalis Arantes

Núcleo de Informática Aplicada à Educação

Universidade Estadual de Campinas

farantes@unicamp.br

Leonardo Cunha de Miranda

Departamento de Informática e Matemática Aplicada

Universidade Federal do Rio Grande do Norte

leonardo@dimap.ufrn.br

M. Cecília C. Baranauskas

Instituto de Computação \&

Núcleo de Informática Aplicada à Educação

Universidade Estadual de Campinas

cecilia@ic.unicamp.br

\section{Tel Amiel}

Núcleo de Informática Aplicada à Educação

Universidade Estadual de Campinas

tamiel@unicamp.br

\section{Maria Cecília Martins}

Núcleo de Informática Aplicada à Educação

Universidade Estadual de Campinas

cmartins@unicamp.br

Resumo Vários projetos ao redor do mundo têm concentrado esforços para inserir em sua prática escolar laptops a serem usados por crianças no modelo 1:1. Neste artigo, apresentamos uma abordagem metodológica para a integração de laptops no ambiente escolar que tem base em conceitos e artefatos da Semiótica Organizacional, explorados de maneira participativa. Descrevemos os primeiros resultados de um Projeto que está sendo realizado com a participação ativa da comunidade de uma escola pública da periferia urbana de Campinas (SP) para a criação de soluções e processos contextualizados. Os resultados das primeiras cinco oficinas semio-participativas, realizadas in loco no espaço da escola, ilustram a viabilidade da proposta metodológica para inserção de laptops XO na escola. Os resultados indicam que a metodologia contribui para antecipar questões particulares ao contexto escolar específico, e pensar soluções de forma compartilhada com os envolvidos mais diretamente com a tecnologia nas práticas da escola, potencialmente promovendo transformações nessas práticas.

Palavras-Chave: inclusão digital, laptops educacionais, semiótica organizacional, design participativo, laptop XO, OLPC.

\begin{abstract}
Projects around the world have focused efforts in integrating laptops into schools to be used by children in a 1-1 model. In this paper we present a methodology for this integration based on Organizational Semiotics concepts and artifacts, explored in participatory workshops. We describe a project underway with the active participation of the school community of an urban school in the city of Campinas (state of São Paulo), focused on fomenting contextualized solutions and processes. The results of the initial five semio-participatory workshops conducted in the school demonstrate the viability of the methods used to negotiate the integration of XO laptops in the school. The results indicate that the methodology contributes to anticipate issues specific to the school context, and think solutions shared among those more directly involved with the technology in the school practices; potentially promoting transformations in these practices.
\end{abstract}

Keywords: digital inclusion, educational laptops, organizational semiotics, participatory design, XO laptop, OLPC.

Recebido: 09 de Dezembro de 2011 / Aceito: 12 Junho de 2012 / Publicado: 04 de Agosto de 2012

DOI: 10.5753/RBIE.2012.20.02.31 


\section{Introdução}

A inserção de novas tecnologias nas escolas tem sido vista por alguns como um problema histórico, para outros como panacéia, e para a maioria como progresso inevitável. Independente das diferenças de opinião, a integração de computadores, em seus vários formatos, continua forte em escolas ao redor do mundo. A compra de laptops e tablets para alunos do ensino básico é talvez o mais recente exemplo do entusiasmo com a inclusão de Tecnologias de Informação e Comunicação (TICs) no ambiente escolar.

Muitos governos ao redor do mundo já distribuíram ou têm como objetivo disseminar laptops para crianças em escolas. Estes números são expressivos como a compra de: 500.000 unidades pelo governo português para uso no Projeto Magalhães; 513.204 unidades no Peru para uso no âmbito do Programa Una Laptop por Niño ${ }^{1}$; e 150.000 unidades do laptop XO no Uruguai [1]. O projeto que, de certo modo, inspirou este movimento One Laptop Per Child (OLPC) ${ }^{2}$ - não tem a escola como espaço privilegiado para o uso de seu laptop, conhecido como laptop XO ou, simplesmente, XO (pronuncia-se "xis-ó").

Há uma expectativa de que a oferta dos laptops para os alunos do ensino fundamental e médio possa romper paradigmas, transcendendo tudo aquilo que já foi realizado até o momento sobre a utilização da Informática na Educação. Essa expectativa é fomentada pela visão de que a utilização destes laptops se diferencia em alguns pontos de projetos já realizados como, por exemplo: i) mobilidade dos equipamentos; ii) possibilidade de utilização dos laptops no ambiente familiar; iii) maior quantidade de beneficiados (projeto com maior escalabilidade); e iv) custo mais acessível.

Por razões políticas e econômicas a grande maioria dos projetos nacionais que incorporam o uso do laptop na prática pedagógica têm como porta de entrada as escolas públicas. No Brasil, a inserção de laptops de baixo custo tem sido feita através de um projeto do governo federal chamado Um Computador por Aluno (UCA) ${ }^{3}$. O UCA começou como um Pré-Piloto em cinco escolas dispersas pelo país, sendo que duas utilizaram o laptop ClassMate (representado pela empresa brasileira Positivo), uma utilizou o laptop Mobilis (representado pela Encore, Índia), e duas outras utilizaram o laptop XO. A segunda fase começou em Maio de 2010 - chamada de fase Piloto - e envolveu a distribuição de 150.000 ClassMate (representado pelo consórcio de empresas:

\footnotetext{
${ }^{1} \mathrm{http}: / /$ www.perueduca.edu.pe/olpc/OLPC_Home.html.

${ }^{2}$ http://www.laptop.org.

http://www.uca.gov.br.
}

CCE/Digibras/Metasys ${ }^{4}$ ) para 300 escolas ao redor do país. Em uma sequência dessa fase, seis cidades receberam laptops para todas as suas escolas públicas (chamadas UCA-total).

Além das duas escolas supramencionadas contempladas com o Pré-Piloto, outras quatro receberam laptops XO através de acordos ou doações. Até meados de 2011, 3.013 unidades do laptop XO já haviam sido distribuídos para os projetos em seis escolas: duas no estado de São Paulo, duas no Rio Grande do Sul, uma em Santa Catarina, e uma no Ceará ${ }^{5}$.

Entendemos que para viabilizar o uso do laptop educacional no cenário escolar é importante que os educadores da escola e do sistema de ensino ao qual ela pertence tenham a oportunidade de refletir sobre as práticas escolares com o uso do laptop, identificar e analisar potencialidades e limitações, e participar das decisões necessárias para que essas práticas possam se concretizar.

O envolvimento da comunidade escolar é, portanto, fundamental para a criação de condições para a gestão das tecnologias na escola de modo a viabilizar o seu uso nas atividades educativas, para fortalecer a interação e a criação de redes internas e externas à escola. Diferente de outros projetos citados nesta seção, o presente trabalho de pesquisa propõe a construção de um modelo participativo de análise de contexto e prospecção de soluções para a implantação do XO na escola e seus impactos fora dela.

A concepção metodológica deste trabalho de pesquisa parte do entendimento da tecnologia situado em um contexto social, com métodos e técnicas advindos da Semiótica Organizacional e do Design Participativo, o que chamados de metodologia semio-participativa [26]. Com essa metodologia buscamos promover a participação ativa da comunidade escolar no processo de design de soluções. É, no entanto, uma iniciativa focada em uma unidade escolar em caráter de projeto piloto. Portanto as devidas precauções interpretativas devem ser tomadas ao extrapolar para contextos de implementação que contemplam toda uma rede de ensino ou um país.

Este trabalho está inserido e apresenta resultados da pesquisa que vem sendo desenvolvida em uma escola da periferia urbana de Campinas (SP): a Escola Municipal de Ensino Fundamental (EMEF) Padre Emílio Miotti. Neste trabalho descrevemos a metodologia semioparticipativa, contextualizada para integração de laptops em ambientes escolares. O Projeto apresentado é a mais recente iniciativa com o laptop $\mathrm{XO}$ no Brasil, que começou em 2010 na escola supracitada através da doação de 512 laptops XO pela Fundação OLPC. Hoje, o Projeto

\footnotetext{
${ }^{4}$ http://www.cceinfo.com.br/uca/.

${ }^{5}$ Dados atualizados podem ser consultados em: http://www.olpc.org.br.
} 
XO-UNICAMP $^{1}$ [24] conta também com o apoio do CNPq. Neste artigo, além da metodologia que está sendo empregada no Projeto, descrevemos também o processo de implementação através das cinco primeiras oficinas realizadas com a comunidade local para compartilhar significados e estratégias iniciais ao Projeto. Utilizando bases da Semiótica Organizacional, membros da comunidade escolar local participam ativamente no processo de criação de soluções para inserção dos laptops em uma escola pública, partindo do contexto de uso da tecnologia na escola e na comunidade.

O artigo está organizado da seguinte maneira: a Seção 2 descreve resultados recentes de projetos realizados ao redor do mundo com o XO e seus desafios associados; a Seção 3 apresenta a metodologia que estamos adotando no âmbito do Projeto XO-UNICAMP; a Seção 4 apresenta os resultados alcançados com a realização das cinco primeiros oficinas semio-participativas; a Seção 5 tece uma discussão acerca dos resultados preliminares; e a Seção 6 apresenta as conclusões finais.

\section{Projetos com o XO e seus Desafios}

Relatórios recentes sobre as duas maiores iniciativas utilizando o laptop XO na América do Sul apontam para inúmeros desafios. No caso do Peru a implementação inicial teve como experiência piloto pequenas escolas rurais. Um estudo preliminar indica que a distribuição dos laptops - uma tarefa complicada considerando as condições geográficas do país - aconteceu com sucesso. Mesmo assim, uma série de barreiras foram ressaltadas: i) a falta de infra-estrutura necessária para fazer uso das máquinas visto que, por exemplo, somente $1,4 \%$ das escolas tinham acesso a Internet e 5\% não tinham eletricidade; ii) falta de capacitação dos professores em exercício; e iii) falta de material pedagógico dirigido às atividades com o computador e relacionado com o currículo escolar [2,3].

Em contraste, a iniciativa XO no Uruguai faz parte de um programa nacional para aprendizado conectado chamado CEIBAL ${ }^{2}$ (Conectividad Educativa de Informática Básica para el Aprendizaje en Línea), tendo o XO como a ferramenta principal para os alunos. O governo começou a distribuição dos laptops em 2007 através de um projeto piloto. Tendo como foco a equidade de acesso a computadores e a Internet, o programa conectou $100 \%$ das escolas públicas à Internet em 2010 e aumentou consideravelmente o acesso a Internet para a população

\footnotetext{
${ }^{1} \mathrm{O}$ nome oficial do Projeto, financiado pelo CNPq, é "XO na Escola e Fora Dela: Uma Proposta Semio-Participativa para Tecnologia, Educação e Sociedade".

${ }^{2}$ http://www.ceibal.org.uy.
}

\section{em geral.}

Apesar do esforço do governo uruguaio, capacitação para professores, manutenção de computadores (quase $20 \%$ das máquinas apresentavam algum problema em um estudo por amostra), o uso significativo dos laptops em sala de aula permanece como preocupações para os organizadores $[4,5]$. Buscando contornar alguns desses problemas, o governo está incentivando a manutenção dos laptops XO através de parcerias locais. Além disso, uma rede não-oficial de simpatizantes do projeto proporciona encontros, auxílio e material de apoio. Estas e outras iniciativas respondem às necessidades imediatas do contexto de implementação.

Percebemos que os problemas identificados nestes três projetos estão alinhados com a literatura histórica sobre a integração de tecnologia nas escolas $[6,7,8,9]$. A larga escala destes projetos e a diversidade do públicoalvo cria uma série de desafios relacionados a conciliar atividades que necessitam de gerenciamento central e atividades que necessitam de maior controle local e autonomia.

Ao analisar a iniciativa da OLPC em vários países, Kraemer et al. [1] enfatizam dois elementos relevantes. Primeiro, que a difusão de inovações requer um entendimento claro de contexto local. Segundo, que inovações em TICs não se sustentam sozinhas. Preocupações com infra-estrutura, desenvolvimento e capacitação de pessoal, e outros aumentam em importância à medida que o grau de inovação cresce.

Apesar de muitas décadas e lições sobre a importância de contexto na integração de novas tecnologias em organizações - como escolas - ainda existem inúmeros desafios para que se considere a participação de grupos locais no desenvolvimento de sistemas de inovação. Como nos aconselha Avgerou [10], arriscamos alienar os beneficiados pelas inovações se trabalhamos somente com "melhores práticas" e imperativos universais ([10], p. 2): "Uma atitude que desconsidera o contexto na exploração das TICs é efetiva em disseminar mensagens poderosas sobre a importância das TICs na economia contemporânea, mas como consequência pode levar a grandes riscos ao frustrar ou direcionar erroneamente esforços locais que tentam fazer sentido e apropriar novas tecnologias" (tradução nossa).

$\mathrm{O}$ contexto desta pesquisa difere de outros projetos que envolvem laptops educacionais em escolas públicas, pois nosso foco é o envolvimento da comunidade escolar para construção de um modelo participativo para a implantação do laptop XO na escola pública e seus impactos fora dela. A metodologia deste trabalho parte do entendimento da tecnologia situado em um contexto social. Espera-se com isso que problemas como infra- 
estrutura, desenvolvimento e capacitação pessoal, bem como possíveis soluções, possam partir da própria comunidade escolar. Na próxima seção, apresentamos a base da metodologia utilizada nesta pesquisa, i.e., os métodos e técnicas da Semiótica Organizacional e do Design Participativo.

\section{Uma Metodologia Semio- Participativa}

A apropriação da tecnologia digital articulada a contextos educacionais deve ser tratada como instrumento de transformação profunda da sociedade. Soluções que façam sentido e que tragam benefícios à sociedade como um todo, requerem uma visão sócio-técnica para tratar o problema e sua solução. Alinhada a essa visão, a metodologia que propomos para implantação de laptops educacionais em escolas públicas - já em curso desde 2010 - é baseada na análise de contexto e prospecção de soluções para uso do laptop dentro e fora da escola, construídas com as partes interessadas, i.e., alunos, profissionais da educação e comunidade.

A concepção e execução da metodologia proposta são apoiadas em bases da Semiótica Organizacional com práticas participativas, sustentadas na compreensão socialmente compartilhada sobre a apropriação tecnológica na vida em sociedade [26]. A Semiótica Organizacional (SO) é entendida como um ramo da Semiótica [11,12] que compreende toda a organização como um sistema de signos e possui o objetivo de estudar estas organizações utilizando os conceitos e métodos da Semiótica.

A Cebola Semiótica é um artefato conceitual da SO que representa qualquer sistema técnico - e.g., o laptop XO - em um contexto social, metaforicamente situado no núcleo de uma cebola, na qual várias camadas de informação influenciam e são influenciadas pelo sistema técnico [13,26]. A Figura 1 ilustra o modelo conceitual da Cebola Semiótica. A camada mais externa, Informal que representa uma subcultura cujos significados, intenções e crenças comuns caracterizam costumes e hábitos organizacionais, e na qual compromissos são estabelecidos, alterados e descartados - pode ser compreendida como um sistema de informação informal. Quando significados e intenções são formalizados em regras, ou seja, em um sistema de informação formal (camada intermediária), esses podem ser modelados e implementados por sistemas de informação técnicos a exemplo dos sistemas computacionais (camada mais interna). A Cebola Semiótica, portanto, explicita que um sistema computacional é apenas parte de uma porção formal de uma organização que, por sua vez, é parte de um sistema informal.

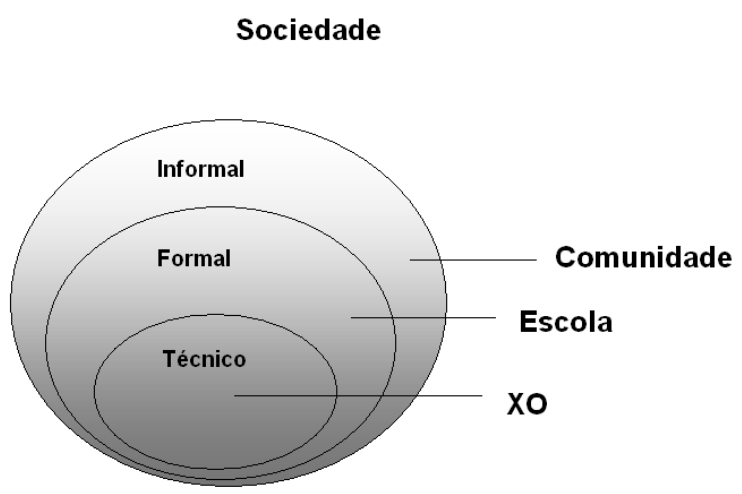

Figura 1: Adaptação do modelo conceitual da Cebola Semiótica para o domínio deste Projeto.

Nesse contexto, a metodologia adotada por este Projeto propõe um movimento feito com as partes interessadas incluindo as camadas informal - família dos alunos e comunidade - e formal - procedimentos e pessoas da escola - em direção a um entendimento socialmente compartilhado para o problema e suas soluções com o intuito de trabalharmos o nível técnico do Projeto potencializando a apropriação efetiva do laptop com propósitos educacionais em larga escala.

Para construir um entendimento do contexto organizacional do Projeto, atravessando as fronteiras das camadas Informal, Formal e Técnica da Cebola Semiótica, artefatos e métodos apropriados são necessários. Os métodos da SO podem ampliar o entendimento sobre quais são as partes interessadas no problema em foco, quais são seus requisitos, bem como as restrições relacionadas à plataforma tecnológica e aos papéis das pessoas envolvidas. Nossa abordagem considera três artefatos para capturar aspectos das diferentes camadas de informação: i) Partes Interessadas; ii) Quadro de Avaliação; e iii) Escada Semiótica. Esses três artefatos são brevemente descritos a seguir:

- Partes Interessadas: permite investigar todas as partes interessadas - os stakeholders - que direta ou indiretamente influenciam ou têm interesses no Projeto sob análise. Para realizar essa análise, as partes interessadas são divididas em quatro grupos: Atores/Responsáveis, Clientes/Fornecedores, Parceiros/Concorrentes e Espectador/Legislador (Figura 2); 


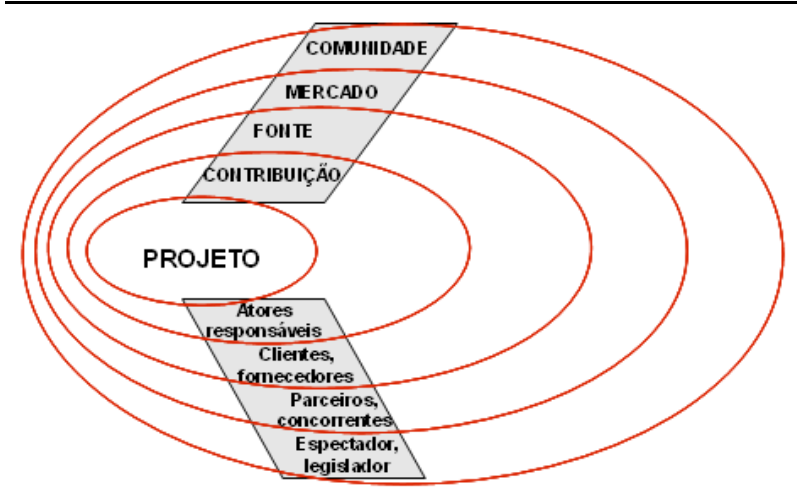

Figura 2: $\mathrm{O}$ artefato Partes Interessadas.

- Quadro de Avaliação: é uma extensão da análise do Partes Interessadas [14] que permite identificar, para cada categoria dos stakeholders, seus interesses, questões e problemas, a fim de antecipar e discutir possíveis soluções (Figura 3);

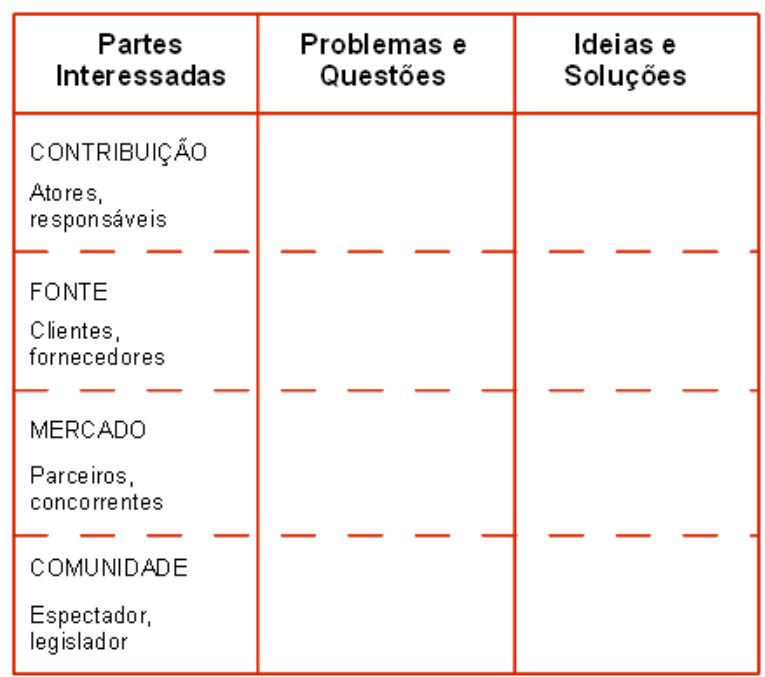

Figura 3: O Quadro de Avaliação.

- Escada Semiótica: enquanto metodologias tradicionais enfatizam questões técnicas (relacionadas ao mundo físico, empírico e sintático), a SO permite analisar outros níveis de relacionamento (semântico, pragmático e social), os quais direta ou indiretamente afetam os aspectos de projeto e uso do sistema (Figura 4).

O uso de artefatos da Semiótica Organizacional em conjunto com técnicas de Design Participativo já foi explorado em trabalhos anteriores de pesquisa em Computação. Podemos citar como exemplos, o uso da abordagem semio-participativa para guiar o processo de elicitação de requisitos [14], o uso da SO como guia para o desenvolvimento de uma arquitetura baseada em serviços para governo eletrônico [30], o estudo de caso sobre a adoção de um processo social para desenvolvimento de sistemas de informação [31], o uso de técnicas semioparticipativas para design inclusivo de sistemas de informação na web [32], dentre outros. Podemos dizer que esses trabalhos representam instâncias do modelo situadas em diferentes projetos; dessa maneira contribuíram com construção da proposta metodológica adotada nesta pesquisa.

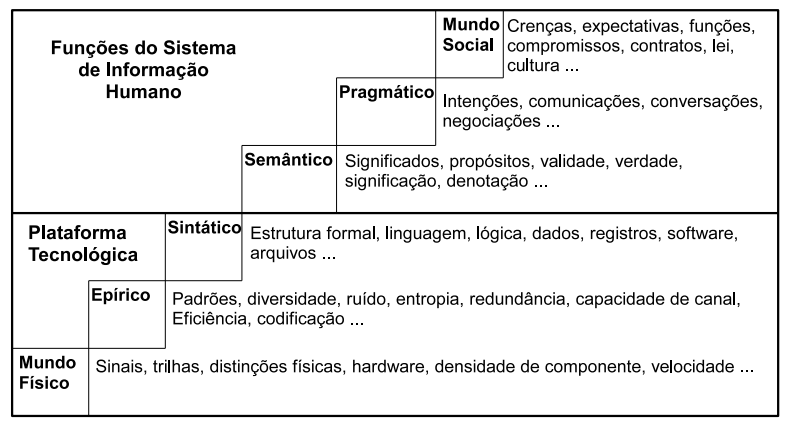

Figura 4: A Escada Semiótica.

Neste trabalho, os artefatos supradescritos foram utilizados em oficinas semio-participativas com as partes interessadas reunindo, em média, 50 pessoas em cada uma das cinco primeiras oficinas do Projeto. Nessas oficinas participaram professores, estudantes, familiares, diretoria e funcionários (Tabela 1).

Tabela 1: Participantes das cinco primeiras oficinas

\begin{tabular}{|l|c|c|c|c|c|}
\hline \multirow{2}{*}{\multicolumn{1}{|c|}{ Papel }} & \multicolumn{5}{c|}{ Oficina } \\
\cline { 2 - 6 } & $\mathbf{1}^{\mathbf{a}}$ & $\mathbf{2}^{\mathbf{a}}$ & $\mathbf{3}^{\mathbf{a}}$ & $\mathbf{4}^{\mathbf{a}}$ & $\mathbf{5}^{\mathbf{a}}$ \\
\hline Alunos da Escola & 5 & 2 & 2 & 0 & 1 \\
\hline Professores & 23 & 25 & 20 & 28 & 22 \\
\hline $\begin{array}{l}\text { Pesquisadores U- } \\
\text { NICAMP }\end{array}$ & 10 & 11 & 12 & 8 & 9 \\
\hline Convidados & 3 & 1 & 0 & 0 & 0 \\
\hline Parceiros SME & 3 & 2 & 1 & 1 & 1 \\
\hline Pais e mães & 2 & 4 & 1 & 0 & 0 \\
\hline $\begin{array}{l}\text { Outros funcionários } \\
\text { da escola }\end{array}$ & 12 & 12 & 12 & 4 & 6 \\
\hline $\begin{array}{l}\text { Participantes do } \\
\text { NIED } 2\end{array}$ & 2 & 2 & 1 & 0 & 0 \\
\hline $\begin{array}{l}\text { Estagiário da Esco- } \\
\text { la }\end{array}$ & 0 & 0 & 0 & 0 & 2 \\
\hline Total & $\mathbf{6 0}$ & $\mathbf{5 9}$ & $\mathbf{4 9}$ & $\mathbf{4 1}$ & $\mathbf{4 1}$ \\
\hline
\end{tabular}

As oficinas foram conduzidas por um grupo interdisciplinar formado por pesquisadores e docentes associados ao NIED, com experiência em educação, psicologia,

\footnotetext{
${ }^{1}$ Secretaria Municipal de Educação da Prefeitura de Campinas.

${ }^{2}$ Núcleo de Informática Aplicada à Educação da UNICAMP.
} 
midialogia, e computação (interação humanocomputador, entre outras áreas). A esse grupo juntavamse outras partes interessadas, incluindo a comunidade escolar (gestores, professores, funcionários da escola e alunos) e pais de alunos; o tamanho e a diversidade da equipe permitiu investigar barreiras e soluções dentro do contexto informal, formal e técnico de maneira plural.

A Figura 5 apresenta uma visão geral, por Oficina, dos papeis assumidos pelos participantes. Como pode ser observado, esse gráfico demonstra como uma diversidade de diferentes partes interessadas está envolvida no processo.

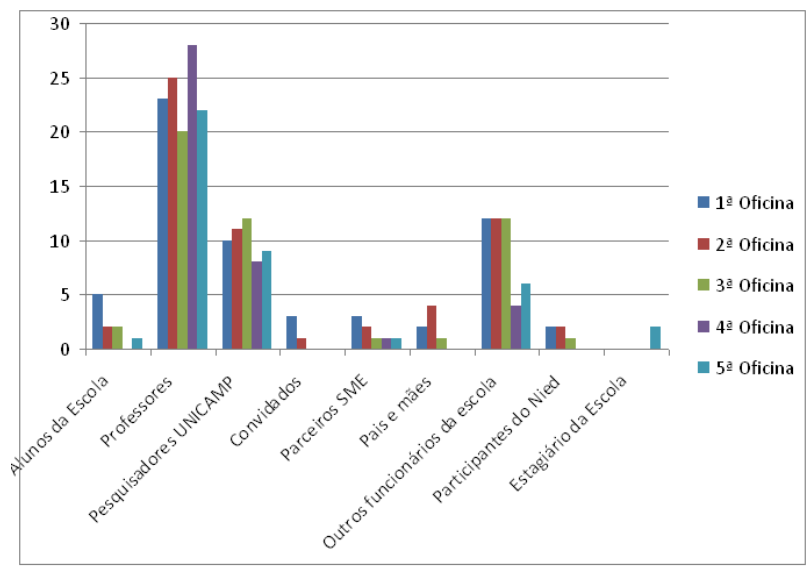

Figura 5: Papéis dos participantes das cinco primeiras oficinas.

Além dos artefatos da SO acima mencionados, as oficinas semio-participativas foram apoiadas por técnicas e dinâmicas do Design Participativo envolvendo os pesquisadores e toda a comunidade escolar. O Design Participativo (DP) $[15,25]$ propõe a participação ativa de usuários em vários momentos de um ciclo de vida de desenvolvimento de um produto tecnológico [16]. No DP, as partes interessadas podem fazer contribuições efetivas que refletem as suas próprias perspectivas e necessidades. Tal participação ativa vai além da visão dos usuários como sendo meras fontes de dados, respondendo a questionários ou sendo observados durante o uso da tecnologia. Muller et al. [15] fazem um apanhado de 61 práticas participativas que podem ser utilizadas durante o ciclo de desenvolvimento de software. Neste Projeto, incorporamos princípios do DP nas atividades semioparticipativas.

A metodologia deste Projeto está relacionada aos princípios do DP ao envolver a comunidade escolar na proposta e análise de soluções para uso dos laptops dentro e fora da escola, considerando aspectos e decisões sobre: apropriação da tecnologia, utilização da tecnologia no contexto educacional, e adaptação do uso da tecnologia à realidade escolar.
Nas próximas seções, descrevemos a realização de oficinas semio-participativas junto à comunidade escolar e como os artefatos da SO foram utilizados na construção do entendimento compartilhado da comunidade sobre o contexto social e organizacional do Projeto. Os dados sobre as oficinas foram compilados através de observações in loco coletadas pela participação dos pesquisadores.

\section{Resultados das Oficinas Semio- Participativas}

Conforme descrito anteriormente, a metodologia adotada neste Projeto endereça a construção de significados de uma maneira participativa, integrada à comunidade escolar (representada em diferentes momentos por estudantes, seus pais, professores e funcionários da escola). Neste trabalho, apresentamos as cinco primeiras Oficinas Semio-participativas (OSp) que foram conduzidas junto à comunidade escolar para tomada de decisões relacionadas ao direcionamento do Projeto.

\subsection{Primeira: Contextualização do Projeto}

A primeira OSp aconteceu em Junho de 2010 e representou o primeiro contato dos pesquisadores com a comunidade escolar envolvida neste Projeto. O objetivo da oficina era contextualizar o Projeto na realidade sóciotécnica da escola utilizando artefatos da $\mathrm{SO}$ em práticas participativas. Apesar do foco da oficina ter sido a discussão do Projeto com a comunidade escolar, a oportunidade do primeiro encontro foi aproveitada para apresentação do laptop XO em uma atividade de exploração livre. A Figura 6 apresenta um momento dessa atividade.

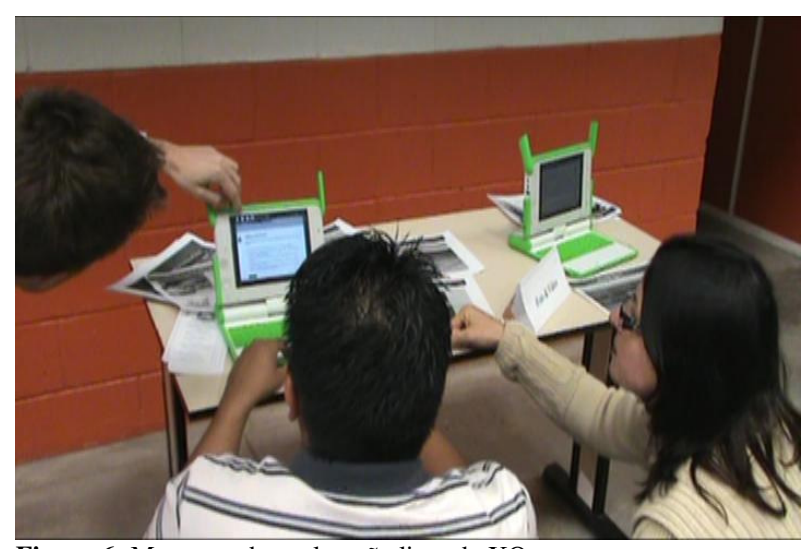

Figura 6: Momento de exploração livre do XO.

De acordo com a metodologia de trabalho proposta, os artefatos da SO foram utilizados por meio de dinâmi- 
cas participativas. O artefato da Figura 2 (Partes Interessadas), como descrito anteriormente, foi utilizado para auxiliar na definição, de maneira abrangente, de todas as pessoas, organizações e entidades que influenciam ou seriam influenciadas pelo Projeto. Em um segundo momento esta informação é utilizada na identificação de possíveis problemas e questões, e ideias e soluções respectivas, com o uso do artefato da Figura 3 (Quadro de Avaliação). A Figura 7 apresenta os participantes da oficina usando esses artefatos.

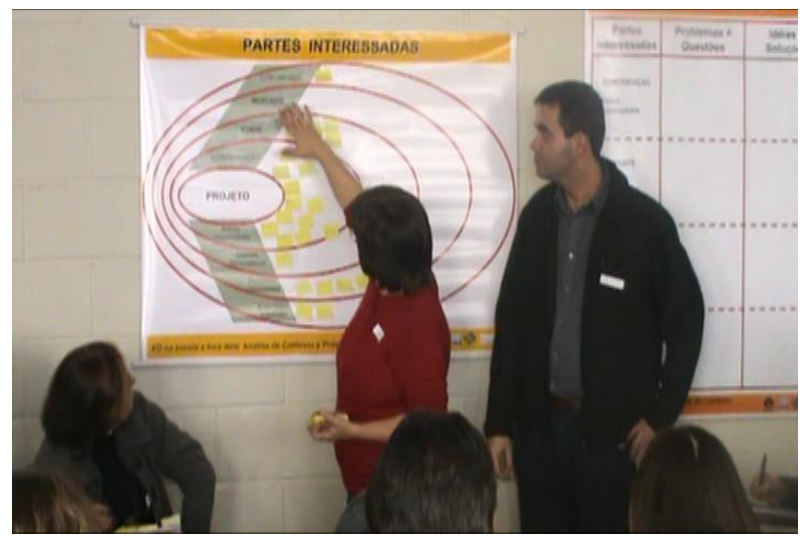

Figura 7: Mediadora colando post-its nos artefatos da SO (ao fundo: Partes Interessadas e Quadro de Avaliação) durante a primeira oficina.

Resultaram da primeira oficina os quadros preenchidos, contextualizando a realidade da Escola, ao mesmo tempo oferecendo proposições de ações para o caminhar do Projeto.

\subsection{Segunda: Atividade "Mão-na-massa"}

A segunda OSp, conduzida em Julho de 2010, representou a primeira oportunidade para os atores escolares utilizarem e explorarem o XO colaborativamente e com a ajuda dos pesquisadores, por meio da atividade "mão-namassa"; vale mencionar que os laptops ainda não haviam sido distribuído às partes, uma vez que esse processo envolvia decisões que seriam discutidas na própria Escola. A atividade começou com uma apresentação de aspectos importantes do laptop incluindo as atividades: ligar o $\mathrm{XO}$; abrir programas; explorar o funcionamento da interface; e explorar dois softwares educativos desenvolvidos pela equipe do Projeto XO-UNICAMP: Caderno Digital [17] e SOO Brasileiro [18].

A análise inicial do contexto organizacional e social do Projeto, obtida por meio dos artefatos da SO utilizados na primeira OSp, apontou a necessidade de situar o uso dos laptops na realidade socio-educacional da comunidade. Por essa razão, depois da atividade "mão-namassa", os grupos foram solicitados a imaginar um cenário educacional - incluindo o laptop - e definir os atores, localização, ações e objetivo do cenário. Como resultado, oito cenários potenciais para uso educacional do XO foram criados, considerando restrições da escola, recursos e objetivos pedagógicos. A Figura 8 apresenta um dos momentos desta oficina.

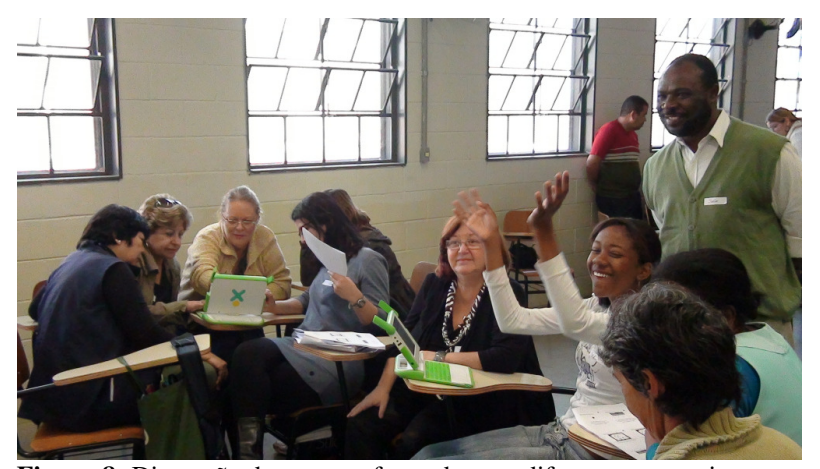

Figura 8: Discussão dos grupos formados por diferentes partes interessadas durante a segunda oficina.

\subsection{Terceira: Propostas para distribuição dos laptops e definição de cenários}

Na terceira OSp, conduzida em Setembro de 2010, foram discutidas propostas apresentadas pela comunidade escolar para distribuição dos laptops e para o logotipo do Projeto. Além disso, foram definidos os próximos passos do Projeto, incluindo os cenários a serem trabalhados no processo de formação dos envolvidos para sua prática pedagógica com os alunos, que incluísse o XO. A Figura 9 apresenta um dos momentos dessa oficina.

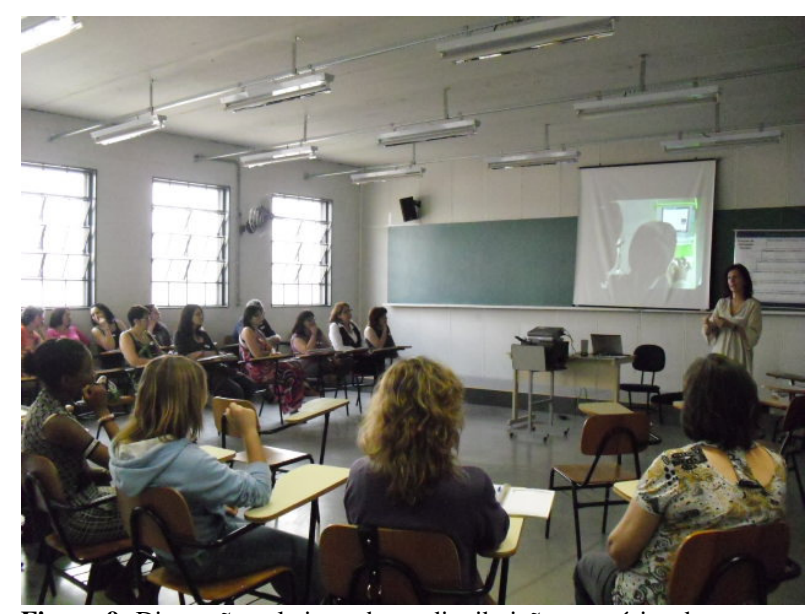

Figura 9: Discussão coletiva sobre a distribuição e cenários durante a terceira oficina.

Entre as oficinas 2 e 3 , os pesquisadores analisaram os oito cenários propostos pela comunidade escolar e os situaram nas camadas da Cebola Semiótica (Figura 10). Quatro deles foram sugeridos para as próximas ativida- 
des: "O Bairro na Cidade" (nível informal); "Explorando o Ambiente da Escola" (nível formal); "Casa do Aluno Consumo" (nível formal); e "Oficina de Locutores de Rádio" (nível técnico).

Durante a terceira oficina, os quatro cenários selecionados foram discutidos. Como a comunidade escolar ainda estava insegura sobre deixar os estudantes levarem os laptops para fora da escola, os professores decidiram não trabalhar o cenário proposto para o nível informal, naquele momento. Ficou decidido que os outros três cenários seriam explorados na oficina seguinte.

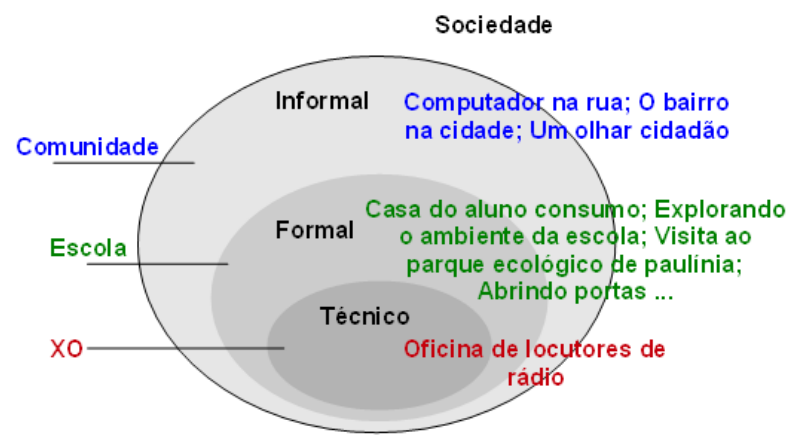

Figura 10: Cenários distribuídos nas camadas da Cebola Semiótica.

Depois da terceira oficina, duas semanas antes da quarta oficina, a escola recebeu seus primeiros 50 laptops (sendo 30 para os professores) do lote total de 512 unidades.

\subsection{Quarta: Formação dos Professores Con- textualizada nos Cenários}

A quarta OSp foi conduzida em Novembro de 2010, com a participação de 21 professores e 8 pesquisadores. Os professores foram distribuídos em três grupos, de acordo com seu cenário de interesse. Cada grupo foi mediado por um pesquisador e apoiado por um facilitador. Descreveremos brevemente a condução do cenário "Casa do Aluno Consumo" como ilustração [27]. Os outros dois cenários foram conduzidos de maneira similar. A descrição desse cenário, que os professores propuseram realizar com as turmas do $6^{\circ}, 8^{\circ}$ e $9^{\circ}$ Anos, foi a seguinte:

"Analisar a composição de produtos industriais que se encontram na casa dos estudantes, especialmente produtos alimentícios. Uma atenção especial deve ser dada à terminologia em Inglês dos produtos. Os estudantes devem criar um documento com a foto do produto, um texto explicativo e a tradução dos termos estran- geiros".

Antes da quarta oficina, os pesquisadores prepuseram um roteiro para cada cenário com o objetivo de antecipar e documentar os possíveis desafios técnicos e pedagógicos que poderiam ser encontrados durante a condução da oficina. Durante a execução do roteiro, os professores foram encorajados a ensinar uns aos outros e a pedir ajuda do facilitador - um pesquisador do Projeto - apenas se não conseguissem realizar a tarefa. Todos os professores conseguiram tirar fotos, criar um documento e importar a foto no documento, bem como explorar o programa de tradução de texto do XO, atividades previstas para o roteiro do cenário "Casa do Aluno Consumo". A Figura 11 mostra os professores da escola durante a realização do cenário.

Ao final da oficina, os professores responderam a um questionário para avaliar sua experiência com o laptop, bem como para avaliar a oficina em si. O questionário foi o mesmo para todos os professores, independente do cenário realizado. O objetivo da avaliação foi identificar aspectos que contribuíam para a execução das atividades do cenário e as que não contribuíam, bem como visualizar possíveis desafios para conduzir os cenários com os alunos.

Um total de 21 professores respondeu ao questionário. Com relação aos cenários, as respostas indicaram a presença de um facilitador como o elemento mais positivo para realização das tarefas; a falta de tempo - cerca de 30 minutos para exploração - foi o mais negativo. A falta de familiaridade com o XO foi apontada como a maior barreira para realizar os cenários com os alunos. Os professores acharam que os alunos terão menos dificuldades que eles para trabalhar com o XO e sugeriram a presença de um "aluno monitor" [19] para ajudar nas atividades com o laptop. Algumas dificuldades técnicas, como falta de carga na bateria e problemas para gravar no pendrive, também foram apontadas. Esses resultados e observações formativas foram de grande valia para os pesquisadores e professores com vistas a conduzir as primeiras atividades com os alunos em situações reais [27].

As respostas ao questionário sobre o uso do $\mathrm{XO}$ pelos professores indicaram que os mesmos exploraram pouco o laptop durante as duas primeiras semanas em que tiveram a posse das máquinas. A maioria explorou o $\mathrm{XO}$ por menos de três horas durante este período $(\mathrm{N}=14)^{1}$. Esse número cai quando perguntamos se usaram o $\mathrm{XO}$ com outros professores $(\mathrm{N}=8)$. Apenas um professor havia usado o XO com um estudante (durante 1 a 3 horas). A maioria havia explorado o XO com outras pessoas - a metade durante 1 a 3 horas $(\mathrm{N}=10)$ e a outra metade

\footnotetext{
${ }^{1}$ Sendo $\mathrm{N}$ o número de professores.
} 
$(\mathrm{N}=10)$ de 4 a 6 horas.

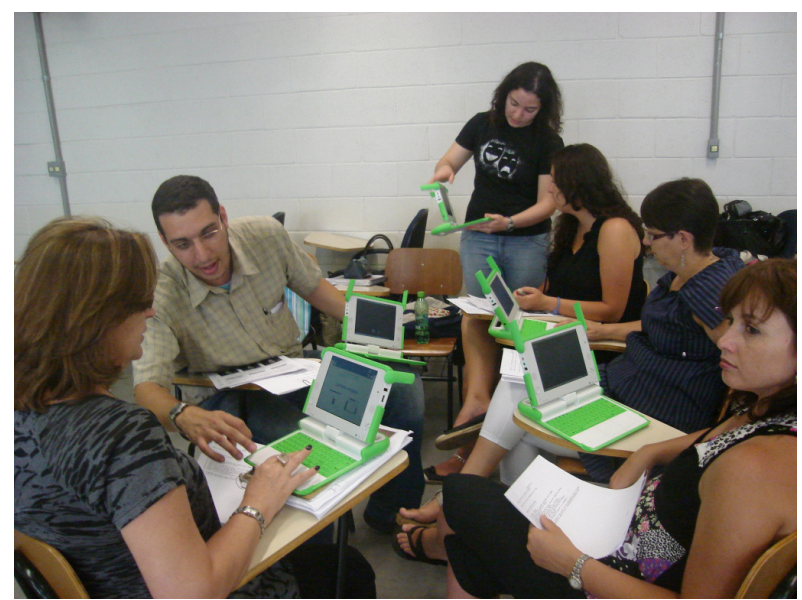

Figura 11: Professores em formação contextualizada nos cenários propostos pela própria escola.

Esses resultados mostram que houve pouca exploração inicial individual e coletiva do laptop no ambiente da escola. Isso indica que a tendência dos professores é explorar o laptop de acordo com suas restrições de tempo, necessidades e demanda. Por outro lado, nossas observações indicam que usar o XO com outras pessoas por exemplo, em casa - aumenta o interesse em explorar e compartilhar o laptop com membros da comunidade. Esperamos que o nível de conforto aumente na proporção de uso do laptop em suas práticas pedagógicas e viceversa.

\subsection{Quinta: Atividades Didático-Pedagógicas com Alunos}

Na semana seguinte à quarta oficina, a escola recebeu mais 120 laptops, os quais foram utilizados para os professores realizarem os cenários com os alunos. A quinta OSp aconteceu em Dezembro de 2010. O objetivo da oficina foi ouvir o relato dos professores sobre o primeiro contato dos alunos com o laptop, registrar suas impressões, dificuldades e expectativas.

Os professores relataram com entusiasmo as atividades dos cenários realizadas com suas turmas (Figura 12). Disseram que os alunos ficaram bastante motivados, inclusive aqueles que apresentavam problemas de disciplina/comportamento. Descrevemos, a seguir, os principais pontos positivos, dificuldades e perspectivas futuras dos professores nesse primeiro momento de uso dos laptops em sala de aula. Resultados detalhados desses usos estão disponíveis em [28,29]:

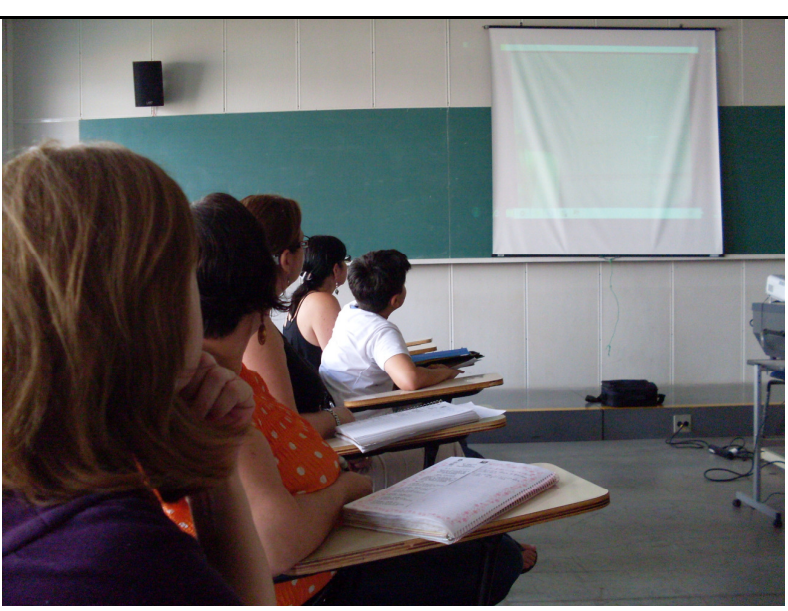

Figura 12: Professores compartilham os primeiros resultados de uso do $\mathrm{XO}$ com seus alunos do ensino fundamental.

- Dificuldades: foram relatados alguns problemas operacionais, tais como a grande quantidade de laptops e o carregamento das baterias dos mesmos. A lentidão do $\mathrm{XO}$, o travamento de alguns programas e a dificuldade para salvar documentos também atrapalharam a realização de algumas atividades. Os professores disseram que o conhecimento sobre as possibilidades da máquina ainda é pequeno e precisa ser melhor explorado.

- Pontos positivos: os professores relataram que as oficinas anteriores e a forma como foram conduzidas pelos pesquisadores foi de fundamental importância para seu envolvimento e motivação. Os professores também apontaram a presença e participação de um estagiário de informática na escola, durante a realização das atividades, como muito importante para seu bom andamento. Ter começado com poucos laptops também facilitou o trabalho. Outro ponto que merece destaque foi a troca de experiência e conhecimento entre alunos e professores nas atividades com os laptops. Os professores começaram a vislumbrar o que pode ser trabalhado com o uso dos laptops.

- Perspectivas: Os professores e a direção propuseram uma sistematização de tempo para planejamento coletivo do trabalho a ser realizado com os laptops no ano de 2011. Eles pretendiam ampliar as propostas de trabalho pedagógico, bem como incluir atividades com as famílias e outros profissionais da escola. A regularização do acesso à Internet na escola e melhorias das condições operacionais para uso dos laptops também foram assuntos em pauta para o ano seguinte. 


\section{Discussão}

As OSp conduziram a um entendimento compartilhado com a comunidade escolar, i.e., professores, alunos, pais, direção e funcionários da escola, para o Projeto, o que permitiu identificar algumas das necessidades e soluções dessa comunidade para o uso dos laptops. Um exemplo disso foi a identificação compartilhada dos cenários, que permitiu vislumbrar questões técnicas e pedagógicas, para iniciar os trabalhos com os laptops em sala de aula.

A realização dos cenários mostrou à equipe de pesquisadores que a apropriação do laptop XO como tecnologia educacional está acontecendo conforme o projeto avança, de uma maneira a articular questões técnicas (na camada Técnica da Cebola Semiótica) e educacionais (nas camadas Formal e Informal da Cebola Semiótica). Por exemplo, a capacitação dos professores está acontecendo em conjunto com as atividades com os alunos e outros interessados, ou seja, não há um momento anterior isolado para formação como observado em outros projetos. Esta abordagem gradual e interconectada pode facilitar o uso efetivo dos laptops por todos.

Além disso, nós identificamos um nível crescente de reciprocidade - apoio mútuo - entre professores e alunos, no que tange o Projeto $\mathrm{XO}$, conforme relatado pelos próprios professores durante a quinta oficina. Pudemos ainda observar, informalmente, que a inserção do XO dentro da escola está transformando relações entre professores e alunos, o que merece maior investigação no futuro. Os relatos também sugerem uma grande troca de experiência e conhecimento entre a comunidade envolvida e os pesquisadores que está fora do escopo deste artigo, mas também merece discussão futura.

Ainda, a inserção de laptops de baixo custo em escolas públicas em nosso país enfrenta problemas e limitações que precisam ser pensados e trabalhados pela comunidade brasileira de Informática na Educação. Muitas vezes, esses problemas vão além da comunidade escolar e da metodologia proposta neste artigo.

A manutenção dos laptops é um problema que ainda não foi resolvido. $\mathrm{O}$ modelo Uruguaio, que funciona de maneira descentralizada poderia ser replicado no Brasil. O modelo utilizado para compras no projeto brasileiro UCA - somente inclui a garantia total do equipamento por 12 meses, conforme consta no Edital do Pregão Eletrônico $n^{\circ} 57 / 2010$ [23]. Considerando as proporções continentais do Brasil, compreendemos que a capilaridade deste modelo poderia ser replicada. Entendemos, ainda, que o apoio poderia ser feito através dos Núcleos de Tecnologia Educacional (NTEs) regionais, empresas locais, ou grupos comunitários, conforme sugerido na primeira oficina. A estrutura institucional das escolas no Brasil muitas vezes dificultam a busca de soluções. Porém, recursos disponíveis diretamente na escola poderiam ser contemplados para tal fim. Tal mecanismo pode também fomentar a capacitação de mão de obra e geração de renda local, ao invés da centralização destes processos nas capitais dos estados. Essa linha de raciocínio, inclusive, está em sinergia com a proposta de desenvolvimento comunitário nucleado pelos os laptops educacionais de baixo custo, já proposto por pesquisadores da comunidade brasileira de Informática na Educação [20].

A falta de infra-estrutura das escolas também pode ser apontada como um problema a ser superado. No caso da escola onde esta pesquisa está sendo realizada, alguns desses problemas, tais como acesso à Internet, carregamento da bateria dos laptops, dentre outros, já foram solucionados. Entretanto, problemas como alocação, instalação e manutenção de servidores esbarram na mesma barreira burocrática relacionada à falta de procedimentos formais que viabilizem o trabalho de uma equipe técnica.

Uma clara limitação do XO é a lentidão do processamento devido a aspectos de hardware, quando comparado ao uso de desktops no laboratório da escola. O carregamento e a execução de muitos aplicativos simultaneamente pode, inclusive, levar ao travamento de processamento e à consequente desmotivação dos alunos e professores, se não orientados às boas práticas de uso desse laptop em particular. Uma análise sócio-técnica desses laptops [21] e uma avaliação da simplicidade de design [22] já foi realizada por nossa equipe de pesquisa em trabalhos anteriores.

O desenvolvimento de novos recursos tecnológicos garante que um laptop se torne obsoleto ou "antigo" depois de 3-5 anos de uso. Tendo em vista os processos políticos e a logística necessárias para a implementação de projetos envolvendo TICs nas escolas brasileiras, em muitos casos os equipamentos, quando finalmente estiverem disponíveis para uso nas escolas, já serão de uma ou duas gerações anteriores aos similares disponíveis no mercado. Uma discussão relevante acerca deste tema é apresentada em [8].

\section{Conclusões e Trabalhos Futuros}

Este artigo apresentou uma iniciativa que visa apoiar a co-construção de um modelo de integração para o laptop XO em escolas públicas. A metodologia adotada no âmbito deste Projeto difere das abordagens que vem sendo empregadas em projetos semelhantes que estão sendo realizados no Brasil e na América Latina, já que o 
situa na realidade sócio-técnica da comunidade escolar e tem sua base na Semiótica e na participação efetiva das partes interessadas ao longo de todo o processo.

A análise preliminar deste Projeto mostra resultados promissores, especialmente no uso de uma abordagem que leva em consideração o contexto local. Esse processo permite que tanto problemas quanto soluções sejam identificados em conjunto entre as agências envolvidas na implementação - neste caso, pesquisadores/Universidade - e os atores escolares, que "recebem" a inovação. Esta complementaridade permite que desafios sejam identificados rapidamente e soluções possam ser organizadas em conjunto com responsabilidade compartilhada, sendo que as soluções são derivadas partindo dos recursos e possibilidades reais do contexto de implementação. Essa abordagem permite que soluções sejam investigadas dentro das reais possibilidades da comunidade. Muitas destas possibilidades podem ser efetivadas imediatamente através de recursos existentes ou via planejamento estratégico. Outras - como melhor acesso a Internet - apresentam maiores desafios que remetem aos atores dos artefatos da $\mathrm{SO}$, investigando parceiros na comunidade ou a necessidade de ação de agentes externos como, por exemplo, governo local ou empresas parceiras.

Sabemos que não existem modelos prontos e comprovados para a implementação de TICs no ambiente escolar. Uma abordagem participativa oferece, no entanto, o potencial para maximizar a destreza e eficácia em alinhar desafios e restrições com iniciativas e soluções. Assim, ao apresentarmos a metodologia e os resultados iniciais, ressaltamos que a mesma abordagem poderia ser empregada para outras tecnologias educacionais, incluindo outros projetos de integração tecnológica, uma vez que o foco do Projeto não é o laptop em si, mas no uso do dispositivo como parte de um esforço informativo e educacional. Outros usos da metodologia em contextos educacionais são reportados em [26].

Acreditamos que os resultados deste trabalho, já no estágio atual, podem contribuir com o intercâmbio de ideias com projetos semelhantes que vem sendo desenvolvidos na América do Sul e ao redor do mundo, estendendo o alcance da discussão dentro e fora da comunidade de Informática na Educação.

Por fim, citamos alguns trabalhos já em andamento que correspondem a desdobramentos desta pesquisa. $\mathrm{O}$ primeiro deles explora a questão da informática comunitária como uma forma de promover o uso do XO entre os pais dos alunos. Outro trabalho aborda a observação participativa das questões afetivas e emocionais em sala de aula, principalmente relacionadas às interações com a tecnologia. Também se encontra em andamento um trabalho relacionado à infraestrutura de servidores para transferência e armazenamento de atividades educacionais. Questões sobre acessibilidade para uso do laptop XO na escola também estão sendo exploradas no contexto desta pesquisa.

\section{Agradecimentos}

Este trabalho contou com o apoio da Fundação de Amparo à Pesquisa do Estado de São Paulo - FAPESP (processo \#2010/11004-9) e do Conselho Nacional de Desenvolvimento Científico e Tecnológico - CNPq (processo \#475105/2010-9). Agradecemos também a Escola Municipal de Ensino Fundamental Padre Emílio Miotti, ao Núcleo de Informática Aplicada à Educação da Universidade Estadual de Campinas (NIED/UNICAMP) e a One Laptop per Child Association, Inc. (OLPC). Agradecemos principalmente aos professores e alunos envolvidos nesta pesquisa.

\section{Referências}

[1] K. L. Kraemer, J. Dedrick, P. Sharma. One Laptop Per Child: Vision vs. Reality. Communications of the ACM. 52(6):66-73, 2009.

[2] A. Santiago, E. Severin, J. Cristia, P. Ibarrarán, J. Thompson, S. Cueto. Experimental Assessment of the Program "One Laptop Per Child" in Peru. Inter-American Development Bank, Washington.

[3] W. Vota. Who is to Blame for OLPC Peru's Failure? An OLPC Intern Viewpoint. http://www.olpcnews.com/countries/peru/who_i s_to_blame_for_olpc_peru.html, Set. 2012.

[4] A. L. Martinez, S. Alonso, Diego Díaz. Monitoreo y Evaluación de Impacto Social del Plan CEIBAL: Metodología y Primeros Resultados a Nivel Nacional. http://www.ceibal.org.uy/docs/presentacion_imp acto_social221209.pdf, Set. 2012.

[5] I. Salamano, P. Pagés, A. Baraibar, H. Ferro, L. Pérez, M. Pérez. Monitoreo y Evaluación Educativa del Plan Ceibal. http://www.ceibal.org.uy/docs/evaluacion_educa tiva_plan_ceibal_resumen.pdf, Set. 2012.

[6] D. Nugroho, M. Lonsdale. Evaluation of OLPC Programs Globally: A Literature Review. http://wiki.laptop.org/images/a/a5/OLPC_Lit_R eview_v4_Aug2010.pdf, Set. 2012. 
[7] L. Cuban. Teachers and Machines: The Classroom Use of Technology since 1920. Teachers College Press, New York, 1986.

[8] T. Amiel. Mistaking Computers for Technology: Technology Literacy and the Digital Divide. AACE Journal. 14(3):235-256, 2006.

[9] M. Trucano. Knowledge Maps: ICT in Education. InfoDev/World Bank, Washington, 2005.

[10] C. Avgerou. The Significance of Context in Information Systems and Organizational Change. Information Systems Journal, 11(1):4363, 2001.

[11] K. Liu. Semiotics in Information Systems Engineering. Cambridge University Press, Cambridge, 2000.

[12] R. K. Stamper. Social Norms in Requirements Analysis - An Outline of MEASUR, In: M. Jirotka, J. Goguen, M. Bickerton (eds.) Requirements Engineering, Technical and Social Aspects. Academic Press, New York, 1993.

[13] M. C. C. Baranauskas, R. Bonacin. Design Indicating through Signs. Design Issues. 24(3):30-45, 2008.

[14] M. C. C. Baranauskas, J. Schimiguel, C. A. C. Simoni, C. M. B. Medeiros. Guiding the Process of Requirements Elicitation with a Semiotic Approach. In Proceedings of the $11^{\text {th }}$ International Conference on Human-Computer Interaction. páginas 100-110, 2005.

[15] M. J. Muller, J. H. Haslwanter, T. Dayton. Handbook of Human-Computer Interaction, chapter Participatory Practices in the Software Lifecycle. páginas 255-297. Elsevier, 1997.

[16] P. Ehn. Usability: Turning Technologies into Tools, chapter Scandinavian Design: On Participation and Skill. páginas 96-132. Oxford, 1992.

[17] D. S. Cardoso, M. C. C. Baranauskas. Um Caderno Digital nos Laptops Educacionais: Proposta Conceitual e Ferramenta. Revista Brasileira de Informática na Educação. 17(2):59-73, 2009.

[18] F. B. O. Silva, R. Romani, M. C. C. Baranauskas. SOO Brasileiro: Aprendizagem e Diversão no XO. Revisa Brasileira de Informática na Educação. 16(3):29-41, 2008.

[19] T. Amiel, G. Morceli. Integração de Alunos Monitores em Salas de Informática: Uma Avaliação. Revista Brasileira de Informática na Edu- cação. 15(1):51-56, 2007.

[20] L. C. Miranda, H. H. Hornung, R. Romani, M. C. C. Baranauskas, H. K. E. Liesenberg. Estendendo a Idéia do Projeto UCA ao Desenvolvimento Comunitário: Reflexão e Estratégias. In Anais do XXVIII Congresso da Sociedade Brasileira de Computação. páginas 107-116, 2008.

[21] L. C. Miranda, H. H. Hornung, D. S. M. Solarte, R. Romani, M. R. Weinfurter, V. P. A. Neris, M. C. C. Baranauskas. Laptops Educacionais de Baixo Custo: Prospectos e Desafios. In Anais do XVIII Simpósio Brasileiro de Informática na Educação. páginas 358-367, 2007.

[22] M. C. Canal, L. C. Miranda, L. D. A. Almeida, M. C. C. Baranauskas. Analisando a Simplicidade do Laptop da OLPC: Desafios e Propostas de Soluções de Design. In Anais do XXXI Congresso da Sociedade Brasileira de Computação. páginas 1250-1264, 2011.

[23] FNDE. Pregão Eletrônico n $n^{0} \quad$ 57/2010. http://www.fnde.gov.br/index.php/pregoes2010/2054-pregao-eletronico-no-572010, Set. 2012.

[24] XO. Projeto XO-UNICAMP. http://www.nied.unicamp.br/xounicamp/, Set. 2012

[25] D. Schuler, A. Namioka. Participatory Design: Principles and Practices. Lawrence Erlbaum Associates, Inc., Hillsdale, 1993.

[26] M. C. C. Baranauskas. Design de Sistemas e o Profissional Reflexivo: Um Modelo SemioParticipativo. In: J. A. Valente, S. Bustamante (Org.) EAD e Reflexão sobre a Prática: A Formação do Profissional Reflexivo. Avercamp, São Paulo, páginas 183-203, 2009.

[27] T. Amiel, F. L. Arantes, L. C. Miranda, M. C. Martins, M. C. C. Baranauskas. A Participatory Approach to Scenario Development for $\mathrm{XO}$ Laptops in Brazil. In Proceedings of the $25^{\text {th }}$ World Conference on Educational Multimedia, Hypermedia and Telecommunications. Páginas 48-57, 2011.

[28] L. C. Miranda, E. C. S. Hayashi, R. Assis, M. C. Martins, M. C. C. Baranauskas. Laptop XO Dentro e Fora da Sala de Aula: Expressão, Diversão e Alfabetização. In Anais do XXII Simpósio Brasileiro de Informática na Educação \& XVII Workshop de Informática na Escola. páginas 1352-1361, 2011. 
[29] L. C. Miranda, R. Assis, M. C. Martins, M. C. C. Baranauskas. Interdisciplinaridade via Laptop da OLPC Experimentada com Alunos do Ensino Fundamental. In Anais do XXII Simpósio Brasileiro de Informática na Educação \& XVII Workshop de Informática na Escola. Páginas 10671076, 2011.

[30] R. Bonacin, C. A. C. Simoni, A. M. Melo, M. C. C. Baranauskas. Organisational Semiotics: guiding a service-oriented architecture for egovernment. In M. C. C. Baranauskas and K. Liu, editors, Proceedings of the First International Conference on Organisational Semiotics, pages 47-58, Campinas, SP, Brazil, 2006.

[31] C. A. C. Simoni, A. M. Melo, M. C. C. Baranauskas. Project Management and Risks Management in Complex Projects, chapter Towards a social-based process for information system development: a case study, pages 177-192. Springer, 2007.

[32] A. M. Melo. Design Inclusivo de Sistemas de Informação na Web. Tese de doutorado. Instituto de Computação, Unicamp, December 2007. 